\title{
Drone-aided Communication as a Key Enabler for 5G and Resilient Public Safety Networks
}

\author{
Syed Ahsan Raza Naqvi*, Syed Ali Hassan*, Haris Pervaiz ${ }^{\dagger}$ and Qiang $\mathrm{Ni}^{\ddagger}$ \\ ${ }^{*}$ School of Electrical Engineering \& Computer Science (SEECS), \\ National University of Sciences \& Technology (NUST), Islamabad, Pakistan 44000 \\ $\{11$ beesraza, ali.hassan\}@ seecs.edu.pk \\ $\dagger$ Institute for Communication Systems (ICS), Home of 5G Innovation Center (5GIC), University of Surrey, UK \\ h.pervaiz@surrey.ac.uk \\ $\ddagger$ School of Computing \& Communications, Lancaster University, UK \\ q.ni@lancaster.ac.uk
}

\begin{abstract}
Wireless networks comprising unmanned aerial vehicles can offer limited connectivity in a cost-effective manner to disaster-struck regions where terrestrial infrastructure might have been damaged. While these drones offer advantages such as rapid deployment to far-flung areas, their operations may be rendered ineffective by the absence of an adequate energy management strategy. This article considers the multi-faceted applications of these platforms and the challenges thereof, in the networks of the future. In addition to providing an overview of the work done by researchers in determining the features of the air-toground channel, the article explores the use of drones in fields as diverse as military surveillance and network-rehabilitation for disaster-struck areas. It also goes on to present a case-study which envisages a scenario in which drones operate alongside conventional wireless infrastructure, thereby allowing a greater number of users to establish a line-of-sight link for communication. This study investigates a power allocation strategy for the microwave base station and the small base stations operating at $28 \mathrm{GHz}$ frequency band. The self-adaptive power control strategy for drones is dependent on the maximum allowable interference threshold and minimum data rate requirements. This study highlights the importance of incorporating the drones in the multi-tier heterogeneous network to extend the network coverage and capacity.
\end{abstract}

\section{INTRODUCTION}

In view of their relatively low cost and high mobility, unmanned aerial vehicles (UAVs) have recently found applications in areas other than those related to the military and reconnaissance. Generally, the term 'UAV' refers to the category of flying vehicles which can include small planes, balloons and drones, that may either be able to maintain flight at altitudes greater than $10 \mathrm{~km}$ (high altitude platforms or HAPs) or below this figure (low altitude platforms or LAPs). As drones have become increasingly accessible to the public, tech companies have been emboldened to explore other avenues where they might prove useful. For instance, vendors such as Amazon have found these UAVs to be sturdy enough for cargo transport. One of the more ambitious projects undertaken by Facebook (called 'Aquila') aims to use swarms of drones to provide 'WiFi in the sky' service to remote areas. This is one of the few promising projects proposed in the recent past that can potentially pave the way in exploiting drones as vehicles of communication for future wireless networks. As such, this article aims to elaborate on miscellaneous issues related to the use of UAVs as network infrastructure. Henceforth, this paper uses the terms 'UAV' and 'drone' interchangeably.
UAV-aided communication is an emerging topic in the field of next generation networks. It is widely believed in academia that UAVs can help shape the public safety networks of the future, whereby drones, owing to their greater mobility, may provide fast service recovery in the event of network infrastructure being damaged. Even if such a scenario does not arise, UAVs may continue to relieve network congestion as their inclusion in the network would allow base stations (BSs) to off-load some of the latter's cellular traffic to the former.

The road to incorporating UAVs in wireless networks, while alluring due to the several benefits that can be reaped, is not without challenges. One of the most important considerations while using UAVs as network infrastructure is their limited energy supply. This has led researchers to work on optimal path trajectories for a limited number of drones required to provide coverage to a certain region-of-interest (RoI). Another interesting aspect of drone deployment is its operational altitude. It will be shown in subsequent sections that the greater the UAV altitude (and hence a larger angle of elevation), the greater the probability of establishing a line-of-sight (LoS) communication link with a user (i.e., a straight line may be drawn from the UAV's transmitter to the user without encountering any obstructions). At the same time, however, increasing user-infrastructure distance causes an increase in the path loss experienced by transmissions. Therefore, there is a need to determine an optimum height that represents a tradeoff between these two quantities.

In view of the UAVs' potential in shaping the future wireless communication paradigm, many facets of their operational details are currently under deliberation in academic circles. One such detail pertains to how these drones may access the wireless channel if they are to fulfill their possible roles in package delivery, traffic surveillance and disaster management. It has been suggested in [1] that cognitive radio technology (CRT) might aid UAVs in dealing with a crowded spectrum, an obvious handicap of operating in an urban environment. A fullduplex operation in this scenario has also been proposed in [2], whereby spectrum sensing (determining the frequency bands not being used by primary, cellular users) and spectrum access (exploiting the unused frequency band for data communication) can be performed simultaneously, which helps conserve precious spectral resources.

Other topics of interest include, but are not limited to, the 
working of UAVs alongside device-to-device (D2D) communicators, network resource management for UAVs and their use in caching popular content in a given RoI. Finally, we have presented a case study envisaging the use of UAVs along with the traditional cellular infrastructures in the future systems such as fifth generation (5G) networks. The main motivation of this study is to investigate the compatibility of UAVs with the traditional cellular infrastructures to enable high data rate requirements while improving the energy efficiency for the $5 \mathrm{G}$ and beyond systems.

\section{Channel Characterization}

The motivation behind utilizing UAVs in futuristic public safety networks is that, given adequate planning, they would be able to provide sufficient connectivity in a disaster-struck area in a short span of time. In this regard, radio frequency (RF) planning helps determine an estimate for the number of drones required for coverage, their optimal altitude and the achievable user rates. If UAVs are to be deployed in cities, it is vital to determine the path loss experienced by signals, given a region's urban landscape. However, an accurate characterization of the channel may be computationally expensive and time consuming, and would defeat the purpose of the drones as agents for swiftly rehabilitating communication links.

Path loss model estimation has undergone several phases of refinement in relevant literature. The authors in [3] attempted to express the LoS probability between a drone and a ground user in terms of the angle of elevation. However, they stopped short of including the shadowing analysis in the system model. The work in [4] expanded the scope of study and categorized communication links into LoS, obstructed line-of-sight (OLoS) and non line-of-sight (NLoS), while basing the setup on a single model city that could not be altered to represent a variety of urban environments.

In view of the rising demand of LAP-based services, Hourani et al. [5] presented a comprehensive statistical path loss model for wireless services provided by LAPs in an urban environment that determined signal blockage primarily on the basis of three parameters: the ratio of the area covered by structures to the total area of the RoI, the number of buildings per unit area and a scalar representing the height distribution of the structures. While the values of these parameters for each of suburban, urban, dense urban and high-rise urban scenarios have been obtained from the standards laid out by the International Telecommunication Union (ITU), the main contribution of the work lies in the fact that it allows RF planning to be performed by exploiting readily-available urban parameters rather than extracting the three-dimensional (3-D) model of the RoI.

\section{PATH PlanNing FOR UAVS}

The operations of a drone are constrained by its limited on-board power. Therefore, a UAV needs to consume power judiciously to fulfill both its flight and communication-related missions. The optimal flight path of a UAV is dependent on the type of application. While it is generally posited that multiple UAVs should be deployed above an RoI if they are to serve as communication infrastructure for ground users, a single UAV is sufficient for data collection from or dissemination to the ground nodes. An example of the first scenario has been studied in [6], wherein the effect of a non-hovering UAV's location with respect to the users on ground on the system data rates was quantified. The UAV was made to track the physical locations of the ground users, with the data rates fluctuating due to the variation in the relative distances between the UAV and the users. However, as stated earlier, energy considerations play a major role in determining drone trajectory in a given scenario. The work done in [7] attempted to fill this gap as it took into account the UAV's propulsion energy consumption while determining its optimal flight path to reach a compromise between achieving higher rates and the energy consumed. Subsequently, a circular trajectory around a ground terminal was proposed which was shown to maximize the energy efficiency (EE) of the UAV. In addition to the aforementioned strategies, cellular coverage may also be achieved by deploying drones that simply hover over an RoI, acting as stationary aerial base stations. Since no path planning technique was required in this case, the primary design problem was restricted to computing the optimal altitude of the UAV. Mozaffari et al. [8] not only determined the best operational altitude for drones, but also determined the optimal number of UAVs that should be used to serve a coverage area. As it has been a recurring trend in most related literature, the authors took the coverage area of a UAV to be represented by a circle. It would be instructive for the reader to note that while a greater number of UAVs does indeed ensure that all ground users can meet their rate demands, it can also lead to an increased interference between the communication links of adjacent drones. This interference can be mitigated by ensuring that each UAV is located such that no two coverage areas overlap with each other. Furthermore, it was demonstrated in the paper that an increase in the number of UAVs in the RoI not only allowed them to transmit at lower powers (and hence increased 'coverage lifetime'), but also lowered the optimal altitude of the drones.

As is the case with terrestrial BSs, ground users can 'associate with' (i.e., use the network resources of) the UAVs that satisfy their quality-of-service (QoS). The authors in [9] tried to exploit this idea to determine the optimal 3-D location of a drone in a wireless network which could maximize the number of users meeting their requisite QoS. They then went on to investigate the effect of changing the QoS values for the ground users in various environments (semi-urban, urban, dense urban and high-rise urban) on the number of users associated with the drone cell. The urban parameters used in this work were the same as those stated in the ITU-R standards.

\section{SuRVEILlance Operations}

As stated earlier, UAVs have historically found application in the military for deployment in many intelligence, surveillance and reconnaissance (ISR) missions. Such scenarios generally involve monitoring locations of interest over a long period of time, with the deployed UAVs relaying information such as images, videos or sensor data to a distant control station.

With time, the role of UAVs has diversified, as they are now increasingly being used for surveying purposes. The study presented in [10] is a case in point. The paper was aimed to help a team of archaeologists determine the likely locations of burial mounds in rural Turkey. After obtaining coarse images of the site through satellite, the researchers used a UAV for a closer inspection of the site. The images gathered through these missions could then be used to perform a 3-D reconstruction 
of the RoI for a more accurate classification of the observed structures. Owing to the limited battery power available to the drone, it could not collect high resolution information from all potential burial sites. Therefore, the authors of the paper proceeded to plan the optimal route for the UAV, such that it visited only the subset of possible burial sites during its flight which maximized the probability of correctly categorizing the sites.

In addition to collecting a sequence of images of an RoI, UAVs may also be deployed in the wild, to obtain live streaming videos of the ground scenes. As drones capturing high definition videos have become increasingly accessible to the consumer, they are expected to play a wider role in making surveillance and search-and-rescue missions easier and safer. The transmission of the live video feed from the UAVs to one or more ground stations is dependent on the wireless channel, which may have uncertain link capacities. Therefore, it is vital for them to adaptively stream the scene, i.e., to adjust the video encoding rate on the basis of link capacity. While some adaptive video streaming techniques are currently being used by online entertainment companies like Netflix, they are not adequate for use in aerial vehicles where mobility is rapid. In [11], the authors presented a system prototype that used a throughput prediction algorithm whereby the system was able to reduce the video freezing time, compared to transmitting a video encoded at fixed bit rates above 6 Megabits per second (Mbps). The adaptive video streaming algorithm also exploited content-aware compression which meant that the UAV only transmitted video frames with certain features of interest.

\section{CRoss-layer Radio Resource Design in UAV-ENABLED NETWORKS}

The operational potency of a UAV is dependent upon two important factors: energy-aware deployment and energy-efficient operation [12]. The former refers to timely replenishment of the power reserves of the UAVs without obstructing the network services provided by them, while the latter is indicative of the measures that may be adopted to minimize the on-board power consumption while achieving the desired objectives. One way to achieve energy-aware deployment is to harness inter-UAV cooperation such that, at any given time, only one UAV can abandon its mission to top up its power reserves. There are instances in literature that have attempted to make UAV flights energy-efficient as well.

\section{A. UAVs and the Internet of Things (IoT)}

The authors in [13] provided an alternative scenario in which drones may be used in wireless networks. Here, the ground users were represented by discrete IoT devices, and their data was received by a swarm of UAVs. The work aimed to minimize the total transmission power from the IoT devices required for their signals to be correctly decoded at the UAV. With each UAV serving a single cluster of devices, the system model also accounted for the mobility of the devices. Consequently, a model based on concepts from optimal transport theory was proposed so that the flight path of each drone consumed the least possible energy.

Extending this interplay between IoT and UAV-enabled connectivity, [14] considered a scenario with two categories of ground users: the downlink users (DUs) that formed a communication link with the UAV, and the D2D users that utilized network resources to communicate directly with each other. The authors investigated cases in which the serving UAV was both static and mobile. Owing to the possible interference between the two types of users, the paper reported a rise, and a subsequent fall in the system sum rate, with an increase in the number of D2D users per unit area. It also showed that the D2D user rates initially fell with an increase in UAV altitude due to a greater interference from the drones, which in turn was caused by an increased probability of establishing LoS links. However, a further increase in drone height caused the D2D rates to rise due to an increase in the path loss.

\section{B. UAV-enabled Communication in Disaster-resilient Networks}

As stated previously, the UAVs' advantage in a communication network is that they can quickly restore limited connectivity in the system by acting as substitutes for the damaged onground infrastructure. In [15], the authors considered a multinode network with each node communicating with a BS. The primary aim of the work was to demonstrate the efficacy of UAVs in maintaining global network connectivity in spite of node failure caused by a disaster. The authors devised a routing protocol for obtaining the optimal path between the node and the BS based on geographical distance and packet reception rates. The connectivity module kept track of all the inactive nodes in the setup. In the event of the system encountering 'dead' nodes, the route from, say, node A to the BS was updated. If, however, a route could not be established between one or more nodes, the deployment module was invoked. Subsequently, a sufficient number of UAVs were deployed in proximity to the disconnected node which was closest to a connected code. Once they started participating in the network, the UAVs needed to be cognizant of their positions relative to each other for tracking and collision avoidance purposes. To this end, the paper proposed a decentralized control strategy which could utilize on-board sensors or inter-UAV communication.

In order to further probe role of UAVs in an emergency situation, we have presented a scenario similar to that of a disaster-struck region, i.e., when all terrestrial BSs are inactive. A total of fifty users are randomly deployed in a particular RoI in which the coverage is provided by two UAVs such that their coverage regions do not overlap. The respective beamwidth angles of two UAVs are represented as $\theta_{1}$ and $\theta_{2}$. The impact of beamwidth angles on the system sum rate and the probability of forming a LoS communication link, $P_{\mathrm{LoS}}$, have been investigated. It is noteworthy that the angle of elevation can be defined as the difference between the right angle and the respective beamwidth angle of the UAV. The coverage radius can be computed using law of triangles depending on the aforementioned elevation angle and the UAV altitude.

The trends for this disaster-struck region are depicted in Fig. 1. It may be observed that beyond a certain value of the beamwidth angle, all the curves of system sum rate exhibit a decreasing trend due to an increase in coverage radius which causes greater attenuation in the communication link between the users and the UAV. Furthermore, reducing $\theta_{1}$ results in a higher system sum rate in comparison to the case where $\theta_{1}=\theta_{2}$. This observation arises due to the reduced coverage radius of one of the UAVs which in turn improves the capacity of the transmission links due to smaller path loss. The $P_{\mathrm{LoS}}$ 
trends versus beamwidth angles are shown for urban scenario environment parameters as defined in [5]. It is obvious from the curve that larger beamwidth angles (i.e. smaller angles of elevation) result in a lower $P_{\mathrm{LoS}}$, if all other parameters are kept fixed.

\section{A Radio Resource Management Perspective in UAV-assisted} Multi-tier Multi-band Heterogeneous Network (HetNet)

UAV aerial base stations can play an important role to improve the network capacity and coverage in crowded areas such as stadiums by offloading the traffic from the cellular infrastructure, with minimal network planning. Similarly, the networks can be densified by deploying numerous small cells along with the macro cells to enhance the achievable data rate and coverage for the future generation systems such as $5 \mathrm{G}$. Traffic offloading through network densification and the use of mmWave technology are key enablers for 5G networks. The main contribution of this work is to investigate the traffic offloading from the cellular infrastructure to the UAVs and its impact on the achievable EE.

In this case study, we have investigated the downlink (DL) transmission scheme in which a high power BS and UAVs, both operating in the microwave $(\mu W)$ band, operate alongside lowpower small BSs (SBSs), which operate on millimeter wave (mmWave) frequency band, as shown in Fig. 2. The users can associate to either $\mu W \mathrm{BS}$, the SBS operating on two different frequency bands $(28 \mathrm{GHz}$ and $73 \mathrm{GHz}$ ) or the UAVs. Based on the user associations, the users can be classified into three different types. The $\mu W$ BS and the SBSs operating at $28 \mathrm{GHz}$ try to maximize the achievable EE of their associated users, the SBSs operating at $73 \mathrm{GHz}$ try to maximize the achievable data rate of their associated users and the UAVs (due to their limited battery capacity) operate at the minimum power level to support the minimum QoS requirements of their associated users. Therefore, this case study demonstrates the viability of using UAVs to serve specific type of users in $5 \mathrm{G}$ and beyond systems.

The authors also proceeded in determining the relationship between the optimal altitude of a UAV (or optimal operating coverage radius that allowed its farthest associated cell-edge user to achieve its desired QoS level) and the achievable system EE. This optimal altitude of a UAV is observed to be directly proportional to $\cos (\theta)$, where $\theta$ denotes the UAV's beamwidth angle, and inversely proportional to the cumulative additional losses experienced by the transmission due to both LoS and NLoS communication links.

Another important contribution of this study is the proposed radio resource management scheme which aims to maximize the system EE. The power control strategy which jointly optimizes the data rate and power consumption has been applied to the $\mu W$ BS and SBS operating at $28 \mathrm{GHz}$ band. As discussed earlier, the UAVs share spectrum with the $\mu W$ BS. Therefore, a self-adaptive power control strategy has been proposed for $\mathrm{UAV}$ s in order to protect the QoS of the users associated with $\mu W$ BS. The scheme considers the following three quantities for power allocation:

- the minimum transmission power to satisfy their user's minimum QoS requirements,

- the power corresponding to the maximum allowable interference threshold that can be tolerated by the users asso- ciated with $\mu W$ BS reusing the same spectral resource, and

- the maximum transmit power of the UAVs.

The power allocated to each associated user is the minimum of these three quantities. More details about the power control strategy for the UAV-assisted, multi-tier, multi-band HetNet, being considered by this paper, are shown in Fig. 3. Furthermore, the system EE in the DL transmission scheme can be defined as the ratio of the sum of all the user data rates to the sum of the power consumed by all the users in the network.

\section{Performance Evaluation}

The system performance has been evaluated in terms of system EE and system sum rate against parameters subject to the users' minimum QoS requirements. Three different power control strategies have been proposed in this considered case study. We have defined the Benchmark approach whereby the $\mu W$ BS transmits at its maximum transmission power to maximize the achievable rate of their associated users. The SBS operating at $73 \mathrm{GHz}$ band is assumed to be in noiselimited regime that's why it always operate a power control strategy to maximize the achievable rate of its associated users under all conditions. The power control strategy which aims to maximize the $\mathrm{EE}$ of the users associated with the $\mu W \mathrm{BS}$ and the SBS operating at $28 \mathrm{GHz}$ band is assumed to be in an interference-limited regime and it is referred as Approach 1. Finally, Approach 2 proposes the power minimization approach, which restricts the $\mu W \mathrm{BS}$ and the SBS operating at $28 \mathrm{GHz}$ to transmit at bare minimum power levels required to fulfill the users' minimum QoS requirements and is indicative of exceptional circumstances such as events of natural disasters and unexpected change in traffic patterns. Due to their limited battery capability, the UAVs' always operate at the power minimization approach as proposed in Approach 2 for all the aforementioned cases.

Fig. 4 is a plot of the system EE versus increasing UAV altitude, for all power allocation techniques. The curves for system EE clearly indicate how Approach 1 outperforms the Approach 2 and the benchmark scheme. It can also be seen that the system EE reaches a maximum value when the UAV altitude is nearly equal to $140 \mathrm{~m}$. Beyond this point, the system EE begins to fall. In fact, at $h=140 \mathrm{~m}$, the EE maximization technique offers a system EE which is $35 \%$ greater than to the case when the UAV is deployed at an altitude of $10 \mathrm{~m}$. It may be seen that at higher UAV altitudes, while $P_{\mathrm{LoS}}$ increases, the path loss also increases due to the increasing UAV-ground user distance. Since the optimal system EE is achieved at approximately $h=140 \mathrm{~m}$ for all three power control strategies, a trade-off exists between the system EE and UAV altitude at this point. Therefore, subsequent simulations have been run for cases where the UAV altitude is $140 \mathrm{~m}$, for a fair comparison.

The variation in system $\mathrm{EE}$ and system sum rate for all power allocation mechanisms with increasing target signalto-interference-plus-noise ratio (SINR), $\tau$, has been studied in Fig. 5. It can be observed here that the Approach 1 is generally superior in terms of system EE to Approach 2 and the benchmark strategy. The fall in system EE seen for the setup employing Approach 1 can be attributed to the fact that the increase in system sum rate is not commensurate with the increase in the power consumed by the network, owing to the 
rising rate demands of the cellular users. In contrast, the system EE of the setup using the benchmark scheme remains invariant as the BS operation is independent of the users' target SINR. It may also be seen from the figure that networks using the Approach 1 and 2 exhibit increasing cumulative user rates for increasing $\tau$.

Table I provides an overview of the achievable system EE versus the target SINR $\tau$ for the three considered power allocation approaches discussed previously in Section V-C. The main motivation is to evaluate the impact on the system performance, in terms of achievable $\mathrm{EE}$, of including a UAVs in the traditional HetNet compromising of high power BSs operating at the $\mu W$ band and the lower power small cells operating at multiple mmWave bands such as 28 and 73 $\mathrm{GHz}$. Table I demonstrates the performance gain in terms of achievable system EE, by utilizing UAVs along with the traditional cellular infrastructure for serving the ground users in an outdoor environment. For example, the advantage of the employing UAVs along with the traditional HetNet can be observed from the fact that at target SINR $\tau=0 \mathrm{~dB}$, the achievable system EE is two times more than the observed performance in the cellular networks without UAVs for all the considered three power allocation approaches.

\section{CONCLUSION}

In this article, we provided a summary of the various aspects of communication using unmanned aerial vehicles (UAVs). Issues related to their deployment and operation in an energy efficient manner were discussed in light of relevant literature. Moreover, a routing protocol for UAVs in disaster-resilient networks was discussed, in addition to the scenario in which UAVs and device-to-device (D2D) communicators occurred simultaneously in a region-of-interest (RoI), as an example of the form that internet of things (IoT)-based networks might take in the future. The latter case exhibited the impact of changing UAV altitudes on the interference experienced by the D2D communicators. We also introduced the concept of using cognitive radio as an enabling technology for using UAVs in fifth generation $(5 \mathrm{G})$ networks.

In the end, we presented a case study which incorporated UAVs in a wireless network equipped with both high and low power base stations (BSs). The prime motivation was to investigate if UAV deployment could satisfy higher achievable data rate demands in a $5 \mathrm{G}$ network utilizing mmWave technology, while maintaining the power consumed by the wireless infrastructure at an acceptable level. The study provided insight to network designers regarding the power control strategies that may be used under different circumstances. We concluded that using UAVs in tandem with conventional cellular network infrastructure helps improve the system EE, while maintaining the quality-of-service (QoS) requirements for the users. This finding bodes well for the use of aerial BSs, with their limited battery capacity, as a key enabler for future $5 \mathrm{G}$ networks with minimal network planning.

\section{ACKNOWLEDGMENT}

This work was supported in part through ESPRC UK Global Challenges Research Fund (GCRF) allocation under grant number EP/P028764/1 and in part by the EU FP7 CROWN project under grant number PIRSES-GA-2013-610524. We would like to acknowledge the support of the University of Surrey 5GIC members for this work.

\section{REFERENCES}

[1] Y. Saleem, M. Rehmani, and S. Zeadally, "Integration of cognitive radio technology with unmanned aerial vehicles: issues, opportunities, and future research challenges," in Journal of Network and Computer Applications, vol. 50, pp.15-31, 2015.

[2] M. Amjad, F. Akhtar, M. Rehmani, M. Reisslein, and T. Umer, "Fullduplex communication in cognitive radio networks: a survey," in IEEE Communications Surveys and Tutorials, 2017.

[3] Q. Feng, E. Tameh, A. Nix, and J. McGeehan, "Modeling the likelihood of line-of-sight for air-to-ground radio propagation in urban environments," in IEEE GLOBECOM, 2006.

[4] Q. Feng, J. McGeehan, E. Tameh, and A. Nix, "Path loss models for air-to-ground radio channels in urban environments," in IEEE VTC, vol. 6, pp. 2901-2905, 2006.

[5] A. Hourani, S. Kandeepan, and A. Jamalipour, "Modeling air-to-ground path loss for low altitude platforms in urban environments," in Proc. of IEEE Global Communications Conference (GLOBECOM), USA, 2014.

[6] F. Jiang and A. Swindlehurst, "Optimization of UAV heading for the ground-to-air uplink," in IEEE J. Sel. Areas Commun., vol. 30, no. 5, pp. 993-1005, Jun. 2012.

[7] Y. Zeng, R. Zhang, "Energy-efficient UAV communication with trajectory optimization," in IEEE Trans. Wireless Commun., vol.PP, no.99, pp.1-1, 2017.

[8] M. Mozaffari, W. Saad, M. Bennis, and M. Debbah, "Efficient deployment of multiple unmanned aerial vehicles for optimal wireless coverage," in IEEE Commun. Lett., vol. 20, no. 8, pp. 1647-1650, Aug. 2016.

[9] R. Yaliniz, A. El-Keyi and H. Yanikomeroglu, "Efficient 3-D placement of an aerial base station in next generation cellular networks," in Proc. of IEEE International Conference on Communications (ICC), May 2016.

[10] H. Ding et al., "A multi-resolution approach for discovery and 3-D modeling of archaeological sites using satellite imagery and a UAV-borne camera," American Control Conference (ACC), USA, pp. 1359-1365, 2016.

[11] X. Wang, A. Chowdhery, and M. Chiang, "SkyEyes: Adaptive video streaming from UAVs," in Proc. of the 3rd Workshop on Hot Topics in Wireless, HotWireless, pp. 2-6, 2016.

[12] Y. Zeng, R. Zhang, and T. J. Lim, "Wireless communications with unmanned aerial vehicles: opportunities and challenges," in IEEE Commun. Mag., vol. 54, no. 5, pp. 36-42, May 2016.

[13] M. Mozaffari, W. Saad, M. Bennis, and M. Debbah, "Mobile internet of things: Can UAVs provide an energy-efficient mobile architecture?," IEEE Global Communications Conference (GLOBECOM), pp. 1-6, USA, 2016.

[14] M. Mozaffari, W. Saad, M. Bennis, and M. Debbah, "Unmanned aerial vehicle with underlaid device-to-device communications: performance and tradeoffs," in IEEE Trans. Wireless Commun., vol. 15, no. 6, pp. 3949-3963, Jun. 2016.

[15] G. Tuna, T. Mumcu, and K. Gulez, "Design strategies of unmanned aerial vehicle-aided communication for disaster recovery," High Capacity Optical Networks and Emerging/Enabling Technologies, pp. 115-119, Turkey, 2012.

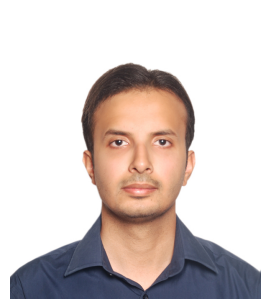

Syed Ahsan Raza Naqvi received his Bachelors in Electrical Engineering degree from the National University of Sciences and Technology (NUST), Pakistan, in 2015. He was previously a part of the Information Processing and Transmission lab at NUST as a research assistant (RA). As a graduate RA, his work primarily focused on the enabling technologies for $5 \mathrm{G}$ networks. His research interests include cooperative communication, millimeter wave technology and device-to-device communication.

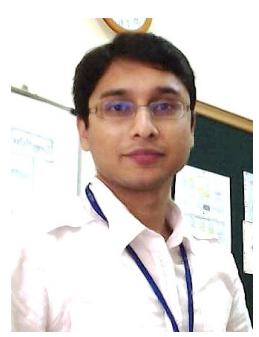

Syed Ali Hassan received Ph.D. electrical engineering from the Georgia Institute of Technology (Georgia Tech), Atlanta, USA, in 2011, M.S. mathematics from Georgia Tech in 2011 and M.S. electrical engineering from University of Stuttgart, Germany, in 2007. Currently, he is working as an Assistant Professor at the National University of Sciences and Technology (NUST), where he is the director of Information Processing and Transmission (IPT) lab, which focuses on various aspects of theoretical communications. 

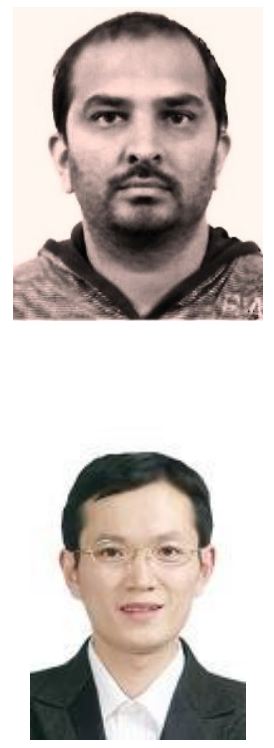

Standards.

Haris Pervaiz received the $\mathrm{PhD}$ degree from the School of Computing and Communication, Lancaster University, U.K in 2016 . He is currently a Research Fellow with 5G Innovation Centre (5GIC) at University of Surrey, UK. From 2016 to 2017, he was an EPSRC Doctoral Prize Fellow at the School of Computing and Communication, Lancaster University, U.K. His main research interests are Green Communications, Control Data Separation Architecture, 5G and Beyond Systems, UAV and Millimeter Wave Communication.

Qiang Ni (M'04-SM'08) received B.Sc., M.Sc., and $\mathrm{Ph} . \mathrm{D}$. degrees in Engineering from Huazhong University of Science and Technology, China. He is a Professor and the Head of Communication Systems Research Group, School of Computing and Communications, Lancaster University, U.K. His research interests are green communications, cognitive radio, 5G, UAV, SDN, IoTs and VANETS. He is a Voting Member of IEEE 1932.1 standard. He was an IEEE 802.11 Wireless Standard Working Group Voting Member and contributor to various IEEE Wireless 
TABLE I: System EE for a given target SINR, $\tau$

\begin{tabular}{|c|c|c|c|c|c|c|}
\hline$\tau[\mathbf{d B}]$ & $\begin{array}{ll}\text { Approach } & \mathbf{1} \\
{[\mathrm{b} / \mathrm{J} / \mathbf{H z}]} & \end{array}$ & $\begin{array}{l}\text { Approach } 1 \text { (no } \\
\text { UAVs) }[\mathrm{b} / \mathbf{J} / \mathbf{H z}]\end{array}$ & $\begin{array}{l}\text { Approach } \\
{[\mathrm{b} / \mathrm{J} / \mathbf{H z}]}\end{array}$ & $\begin{array}{l}\text { Approach } 2 \\
\text { (no } \quad \text { UAVs) } \\
{[\mathrm{b} / \mathbf{J} / \mathbf{H z}]}\end{array}$ & $\begin{array}{l}\text { Benchmark } \\
\text { approach } \\
{[\mathrm{b} / \mathrm{J} / \mathrm{Hz}]}\end{array}$ & $\begin{array}{l}\text { Benchmark } \\
\text { approach (no } \\
\text { UAVs) }[\mathrm{b} / \mathrm{J} / \mathbf{H z}]\end{array}$ \\
\hline 0 & 301.06 & 134.61 & 171.35 & 85.59 & 76.41 & 35.71 \\
\hline 2.5 & 272.95 & 119.2219 & 166.07 & 78.50 & 76.41 & 35.71 \\
\hline 5 & 240.55 & 103.39 & 158.03 & 70.10 & 76.41 & 35.71 \\
\hline 7.5 & 206.51 & 85.83 & 143.75 & 62.2 & 76.41 & 35.71 \\
\hline 10 & 175.46 & 72.06 & 131.69 & 55.22 & 76.41 & 35.71 \\
\hline 15 & 133.65 & 56.25 & 112.87 & 47.08 & 76.41 & 35.71 \\
\hline 20 & 110.07 & 47.14 & 101.73 & 42.24 & 76.41 & 35.71 \\
\hline
\end{tabular}




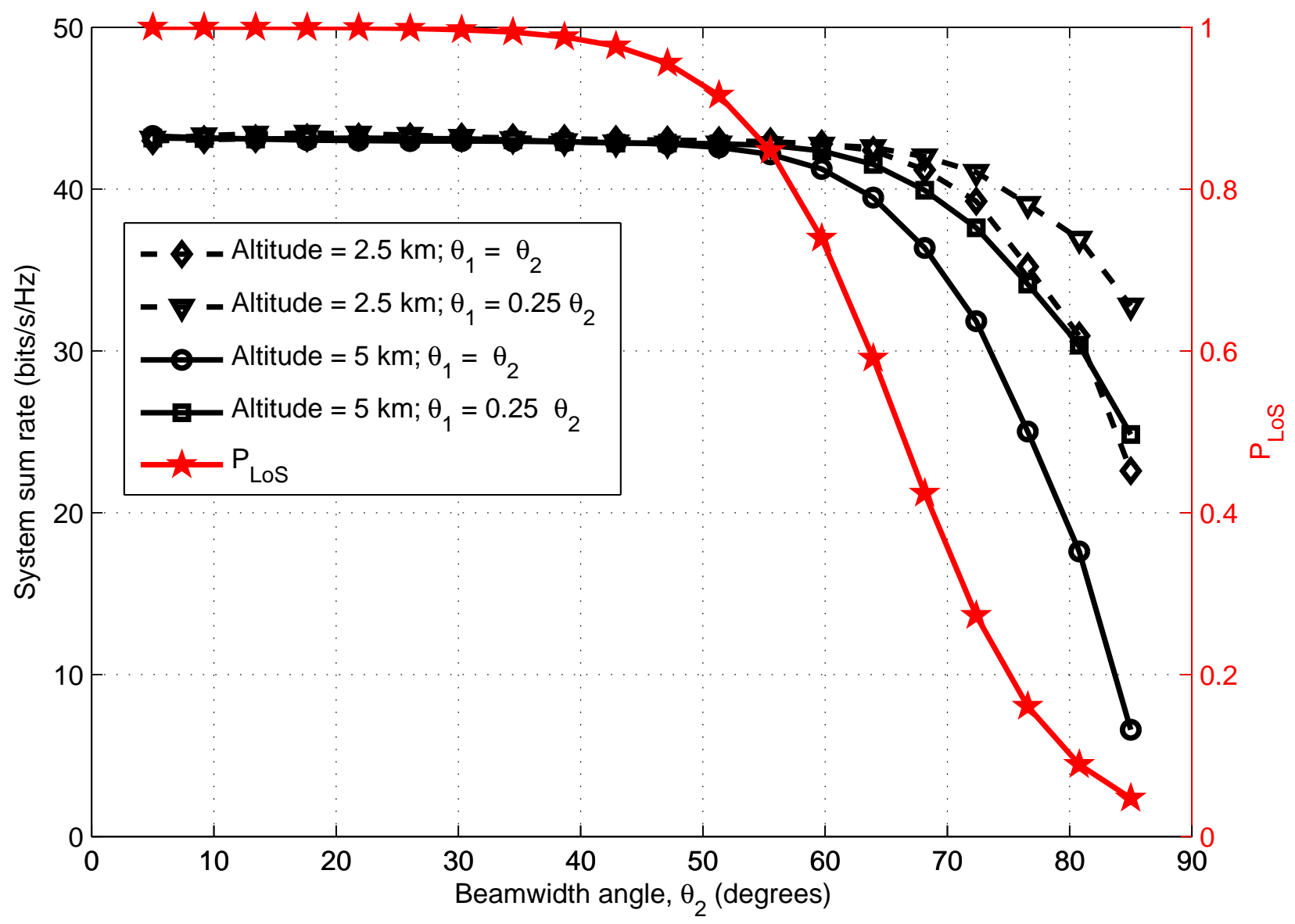

Fig. 1: System sum rate and $P_{\text {LoS }}$ versus beamwidth angle.

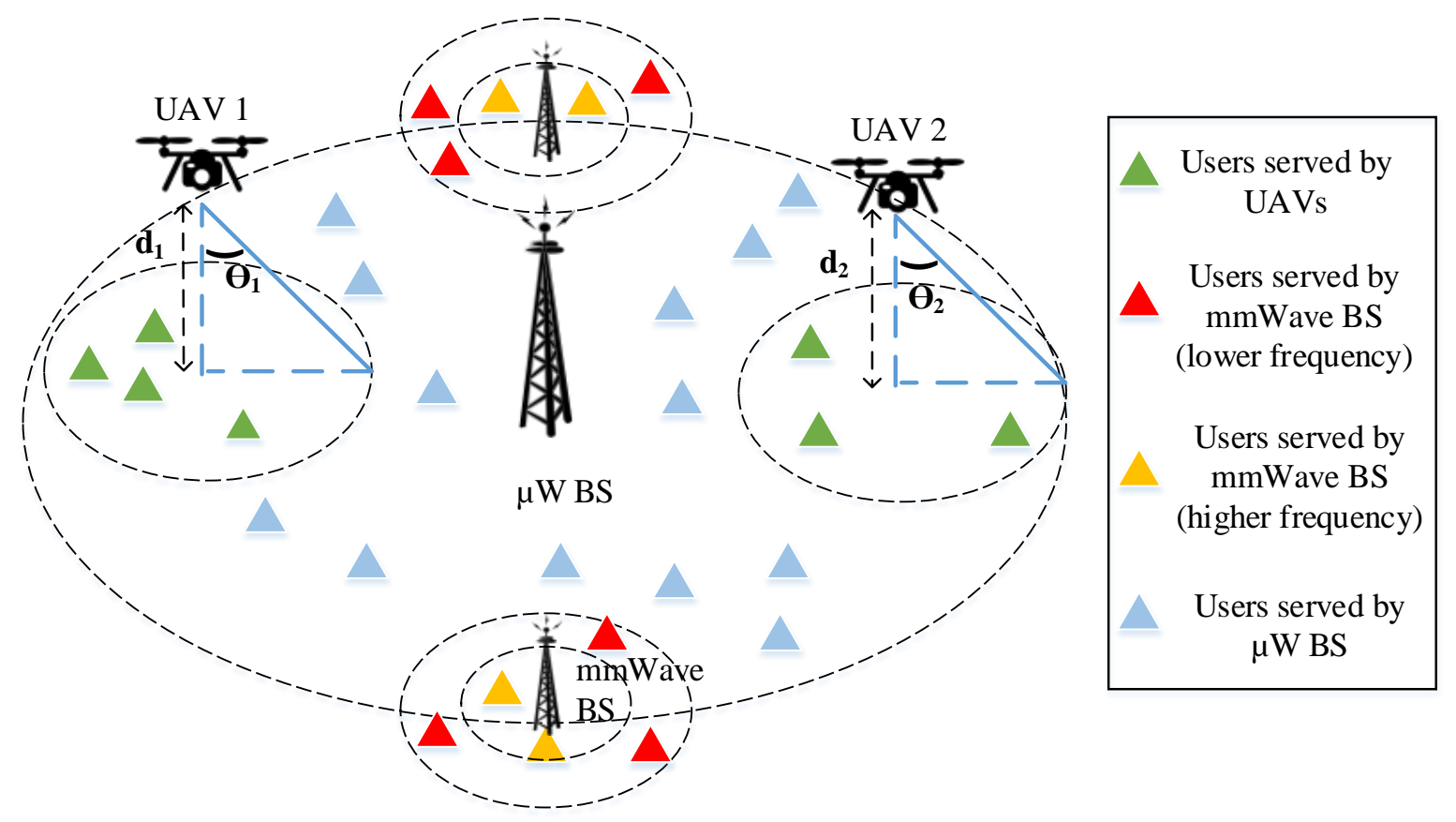

Fig. 2: System Model of UAV-assisted 5G Cellular Infrastructure Case Study. 


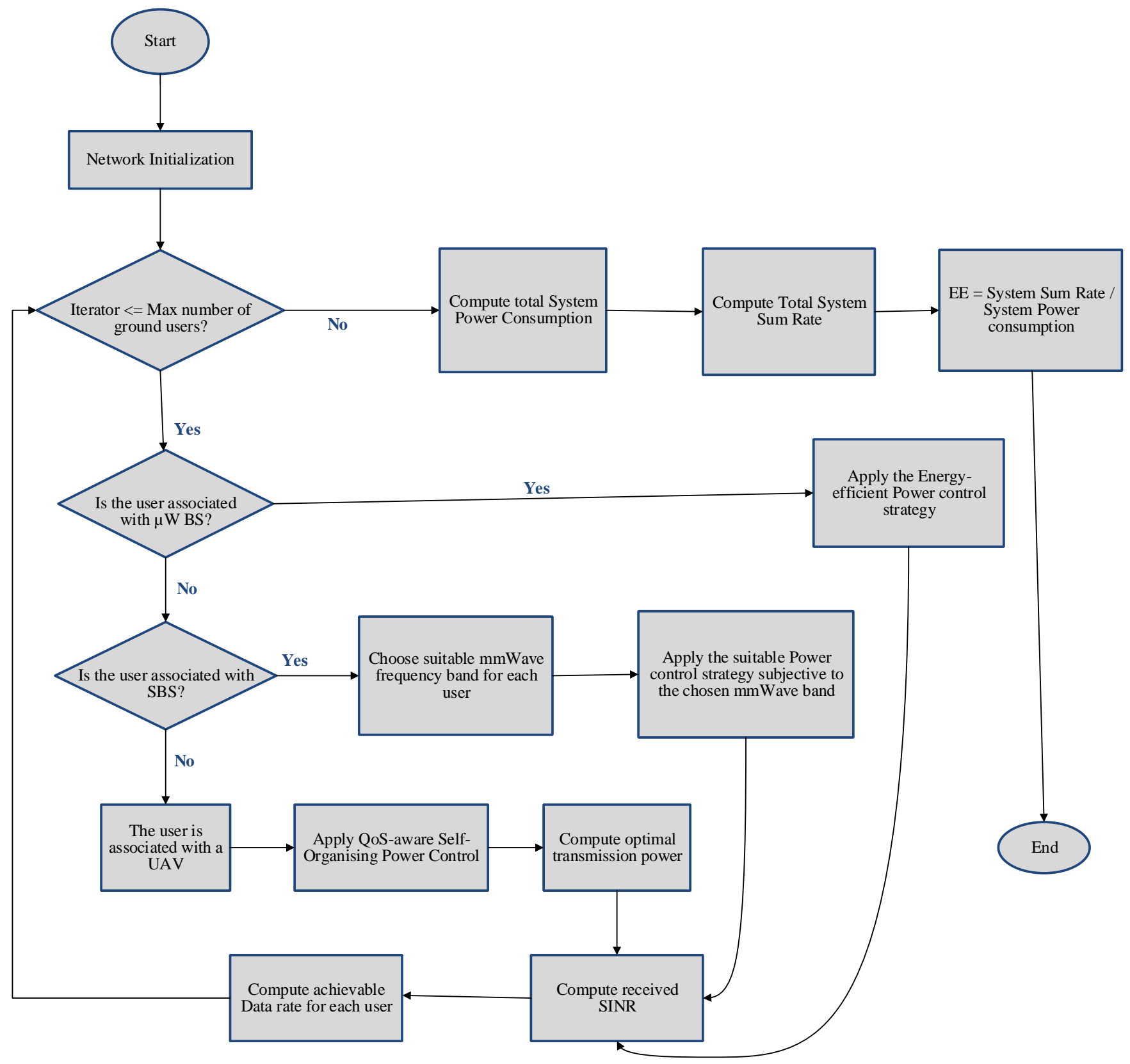

Fig. 3: Implementation flow of the case study. 


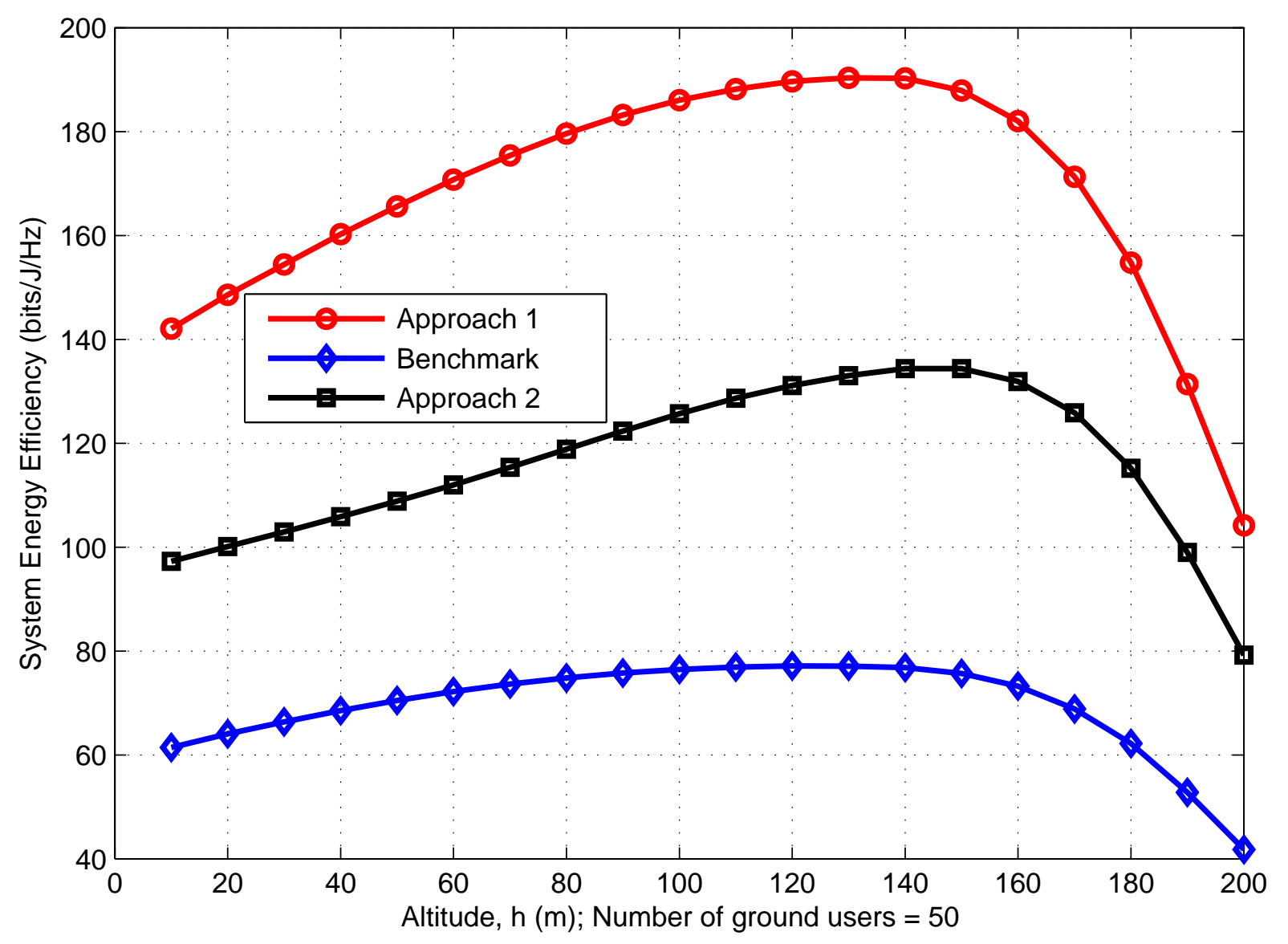

Fig. 4: System EE versus UAV altitude, $h$. 


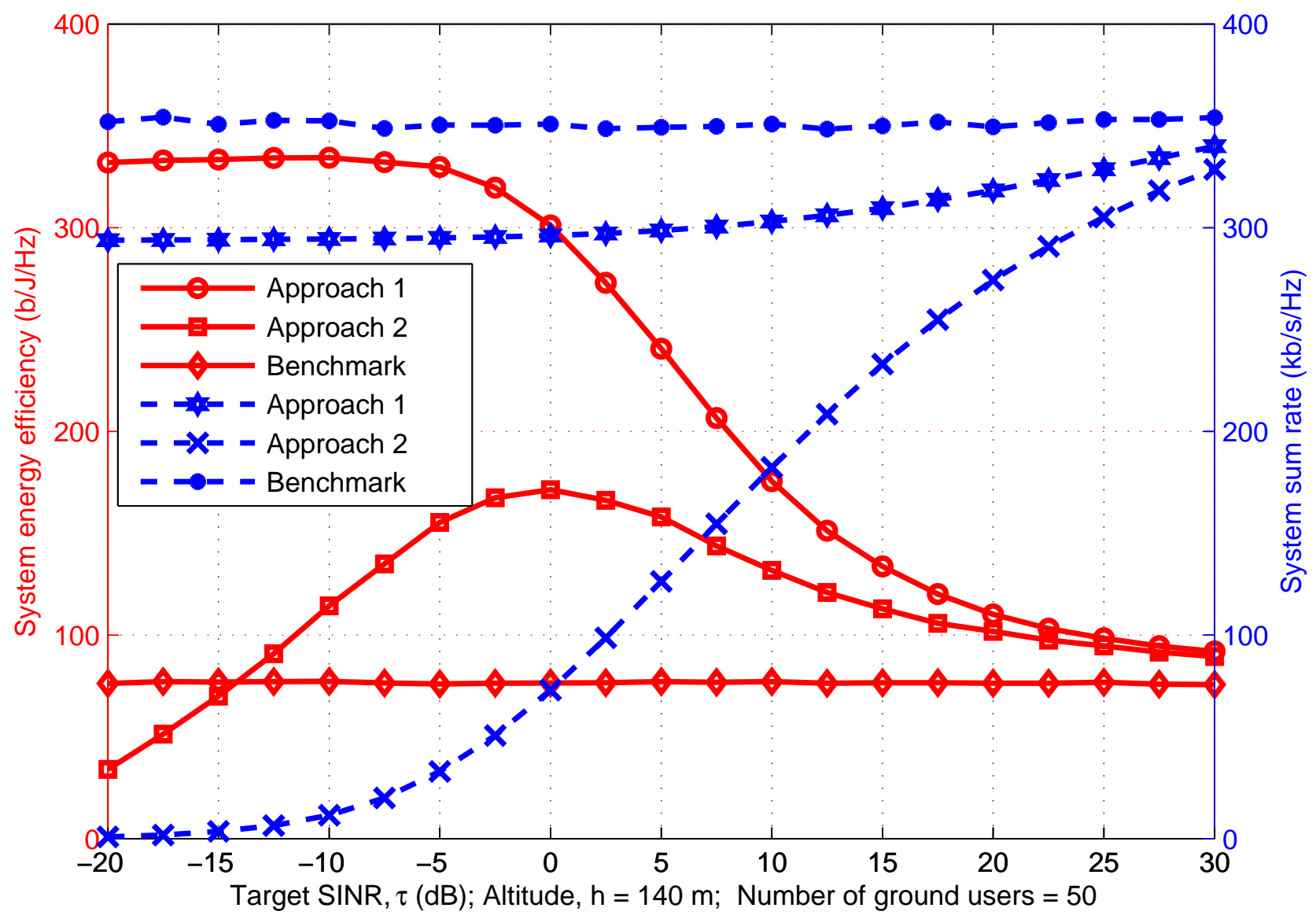

Fig. 5: System sum rate and system EE versus target SINR, $\tau$. 